\title{
Damage Detection of Closed Crack in a Metallic Plate Using Nonlinear Ultrasonic Time Reversal Method
}

\author{
Wang Zhang, Weiliang Wu, Xiaoyi Sun, Li Xiao, and Wenzhong Qu \\ Department of Engineering Mechanics, Wuhan University, Wuhan 430072, China \\ Correspondence should be addressed to Wenzhong Qu; qwz@whu.edu.cn
}

Received 2 August 2013; Accepted 17 October 2013

Academic Editor: Junhong Park

Copyright (c) 2013 Wang Zhang et al. This is an open access article distributed under the Creative Commons Attribution License, which permits unrestricted use, distribution, and reproduction in any medium, provided the original work is properly cited.

Initial cracks in metallic structures incline to be closed at rest. Such incipient damage generally fails to be detected and located with traditional linear ultrasonic techniques because ultrasonic waves penetrate the contact area of the closed crack. In this paper, an imaging algorithm based on nonlinear ultrasonic time reversal method is proposed to detect closed cracks in aluminum plates. Two surface-bonded piezoelectric transducer arrays are used to generate, receive, and reemit ultrasonic wave signals. The closed crack is simulated by tightening a bolt on the aluminum plate. By applying large amplitude excitation voltage on the PZT transducers, the closed crack could be opened and closed. The transmitted waves recorded by PZT array contain nonlinear components, the signals are time reversed and emitted back, and the tone burst reconstructions are achieved. The linear reciprocity and the time reversibility break down due to the presence of the nonlinear components. The correlation coefficient between the original excitation signal and the reconstructed signal is calculated to define the damage index for individual sensing path and is used to develop an imaging algorithm to locate the closed crack on the plate. The experimental results demonstrate that incident wave signals and their reconstructed signals can be used to accurately detect and locate closed cracks.

\section{Introduction}

In mechanical, aerospace, and civil infrastructures, metallic components made of aluminum are ubiquitous. Failures in metallic structures are often caused by closed cracks developed due to fatigue. In practice, closed cracks usually cannot be avoided. Thus, there is a need in nondestructive inspection for detection of such incipient damage. The closed crack stays in contact and presents the same as linear damage at rest, and only when the excitation overcomes a threshold, the closed crack can be opened and produces nonlinear response, so it is hard to detect closed crack with traditional linear ultrasonic technology. Since the nonlinearity signatures are much more sensitive to small damage features than the measurement of the variations of linear elastic properties [1], it is a very promising field of research to detect nonlinear scatterers in metallic structures using nonlinear ultrasound. However, the nonlinear response is usually small and hard to be detected, and hence imaging the closed crack is a challenging problem. Recently, more and more attentions had been drawn to the damage detection with application of time reversal wave signals. This technique uses the reconstruction property of the time reversal procedure; that is, an original wave can be reconstructed at its source point if its forward wave recorded at another point is time reversed and emitted back to the source point. Time reversal method has been used to focus Lamb wave energy to detect flaws or damages in plates by Ing and Fink $[2,3]$. Park et al. investigate the time reversal process of Lamb wave signals which are transmitted and received by piezoelectric transducers bonded on plate-like structures [4]. Xu and Giurgiutiu address multimode Lamb waves by developing a theoretical model for the analysis of piezoelectric-wafer-active-sensors- (PWAS-) related Lamb wave time reversal based on the exact solutions of the Rayleigh-Lamb wave equation [5]. Ulrich et al. conducted Lamb wave time reversal experiments to detect the damage simulated by steel block on plate [6]. The time reversal reconstructed wave differs from the original wave. However, this block damage is a linear scatterer; the reconstructed signal should be close in shape to the original wave. If the signals contain nonlinear contributions, a nonlinear scatterer is said to be located in the position of the focal 


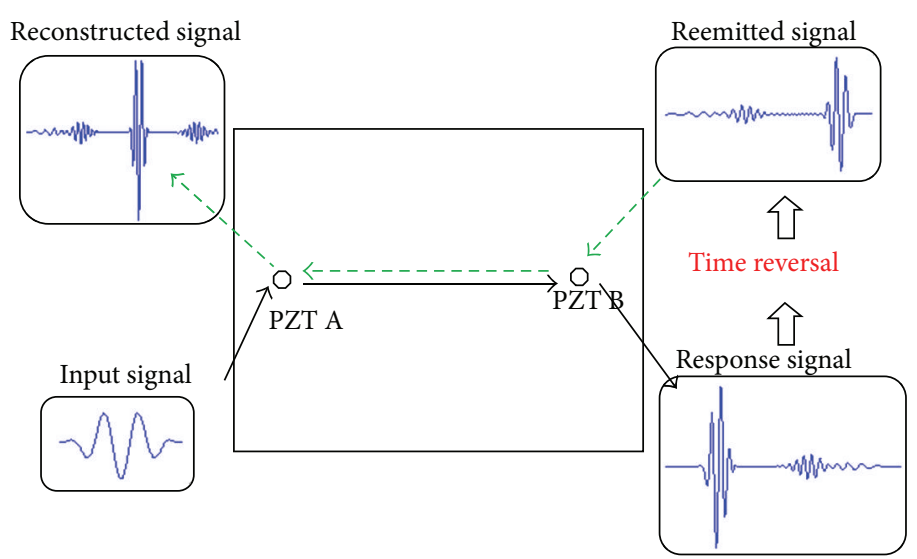

Figure 1: A sketch map of the Lamb wave time reversal process [4].

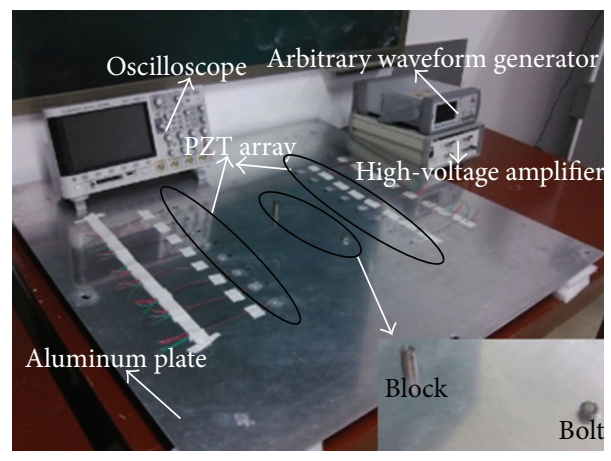

Figure 2: Experimental setup.

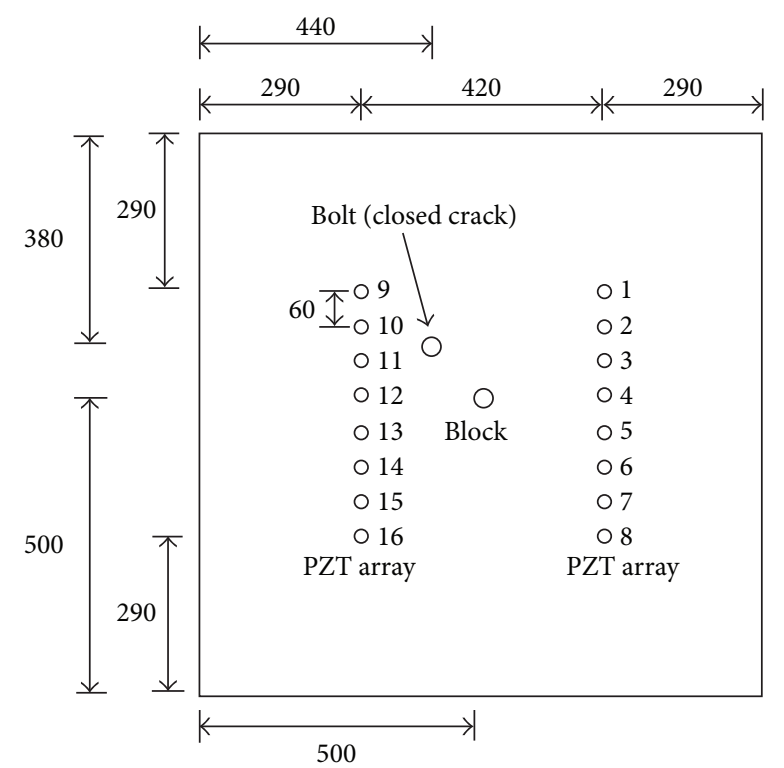

FIGURE 3: Geometry of plate showing the PZT array and damage position. 


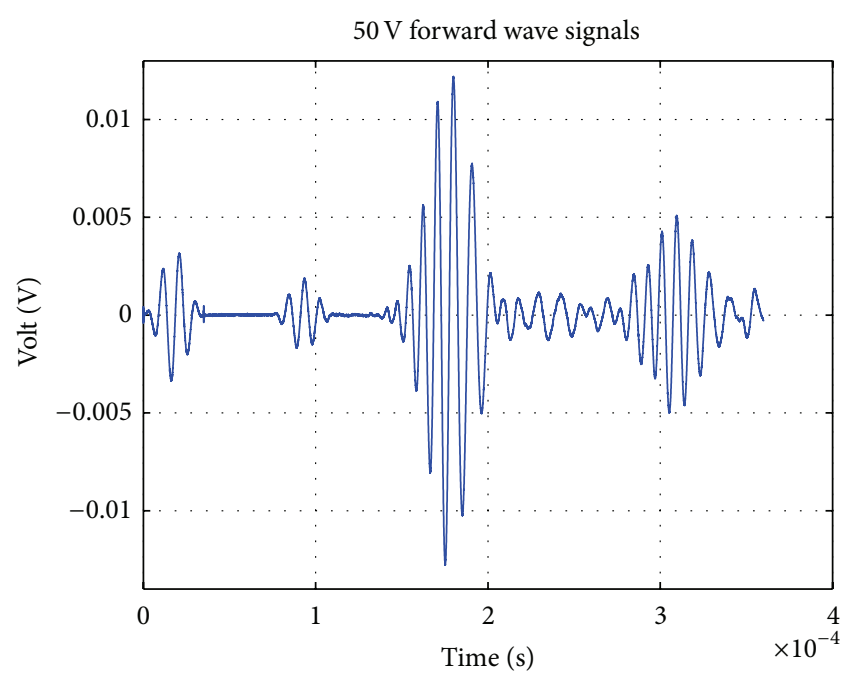

(a)

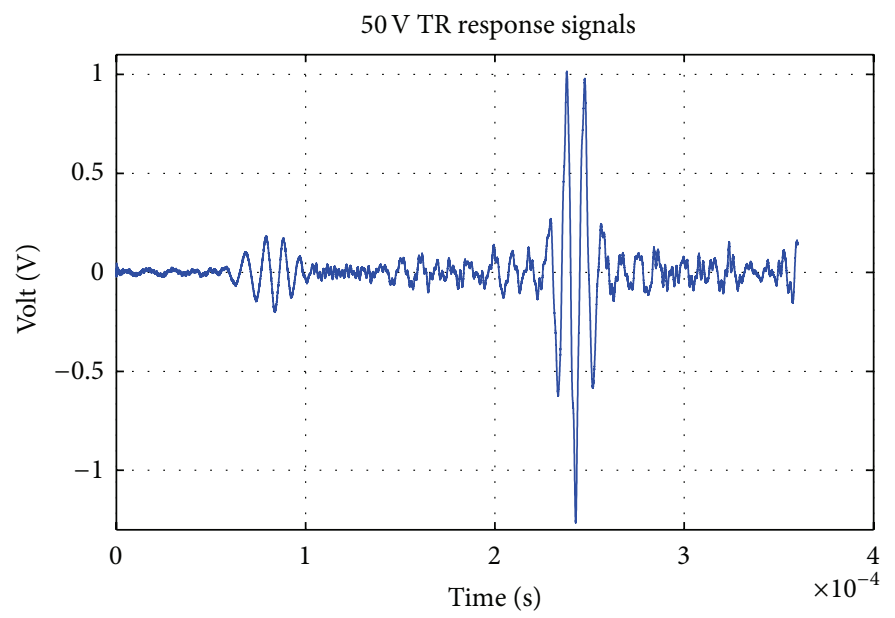

(c)

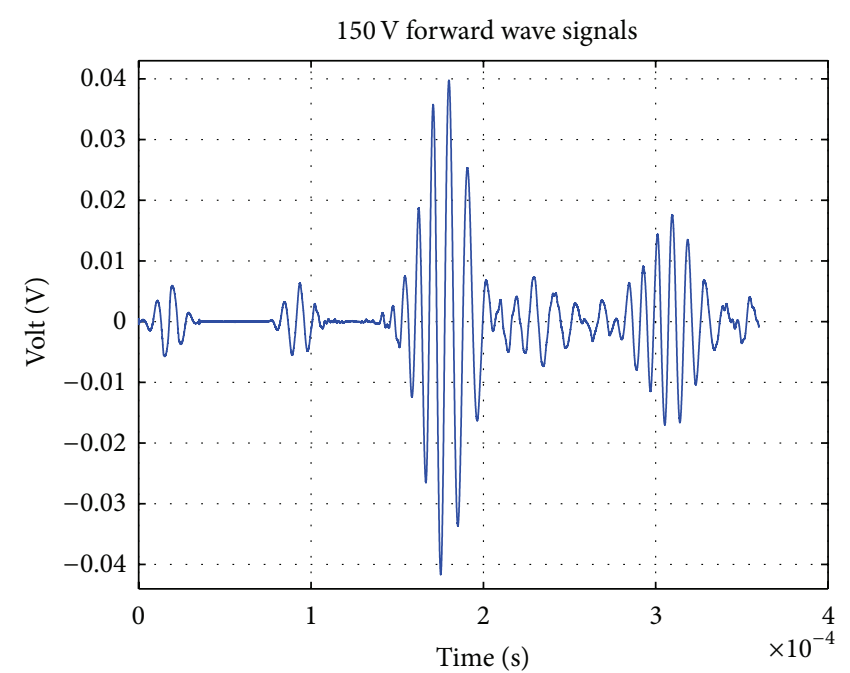

(b)

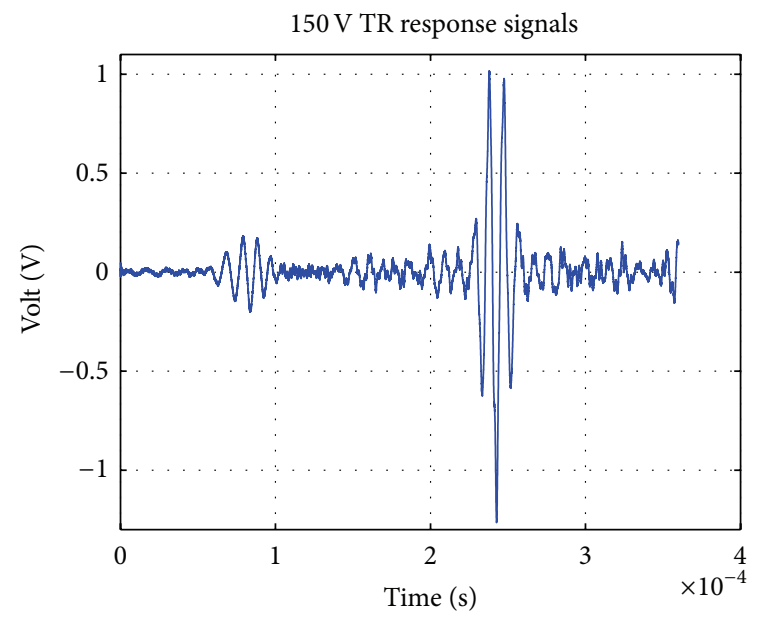

(d)

FIGURE 4: Experimental waves in Lamb wave time reversal procedure for the path P7-16: (a) forward wave after $424 \mathrm{~mm}$ (50 V excitation), (b) forward wave after $424 \mathrm{~mm}$ ( $150 \mathrm{~V}$ excitation), (c) reconstructed wave (50 V excitation), and (d) reconstructed wave (150 V excitation).

spot. Although the Lamb wave time reversal technique has been attempted experimentally and shows its effectiveness for detecting certain types of damage, the experiment of imaging closed crack using Lamb wave time reversal has not been reported yet.

This paper starts with a description of the time reversal method. The main part covers the use of the time reversal nonlinear ultrasonic wave signals method for detecting and imaging damage areas with closed crack in aluminum plate. Experimental studies are carried out to examine the proposed method. Finally, this paper concludes with a brief summary.

\section{Time Reversal Process of Wave Signals}

The principle of the time reversal process in a twodimensional plate is illustrated in Figure 1, where a tone burst is applied to transducer A functioning as a transmitter (step 1 ), activating a wave signal that is captured by transducer
$\mathrm{B}$ acting as a receiver (step 2); the captured signal is time reversed in the time domain (step 3 ) and reapplied to transducer $\mathrm{B}$; then, the wave signal at transducer $\mathrm{A}$ is recorded and time reversed as a reconstructed signal of the original one (step 4).

Based on Figure 1, when a tone burst signal $V_{\mathrm{A}}(\omega)$ at a central frequency $\omega$ is applied to transducer A, it activates a wave signal that propagates within the plate. The wave energy generated by transducer $\mathrm{A}$ is defined by

$$
E_{\mathrm{A}}(\omega)=k_{\mathrm{A}}(\omega) V_{\mathrm{A}}(\omega),
$$

where $k_{\mathrm{A}}(\omega)$ is the electromechanical coefficient of transducer $\mathrm{A}$. The wave signals captured by transducer B can be defined by

$$
V_{\mathrm{B}}(\omega)=k_{\mathrm{B}}(\omega)^{-1} G(\omega) k_{\mathrm{A}}(\omega) V_{\mathrm{A}}(\omega),
$$

where $G(\omega)$ is the structural transfer function of the plate from A point to $\mathrm{B}$ point. $k_{\mathrm{B}}(\omega)$ is the electromechanical coefficient of transducer B. 


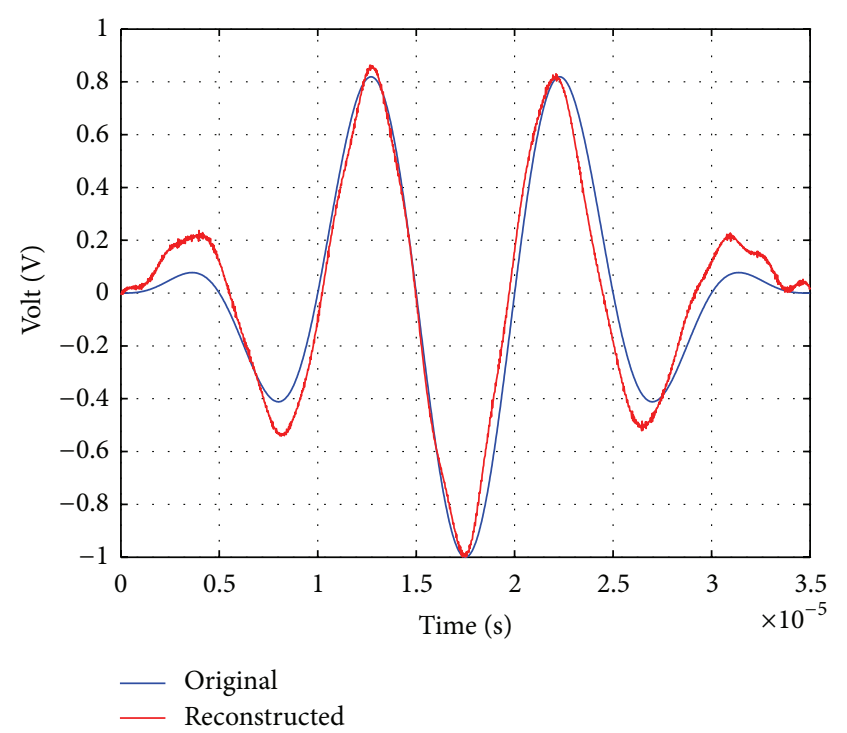

(a)

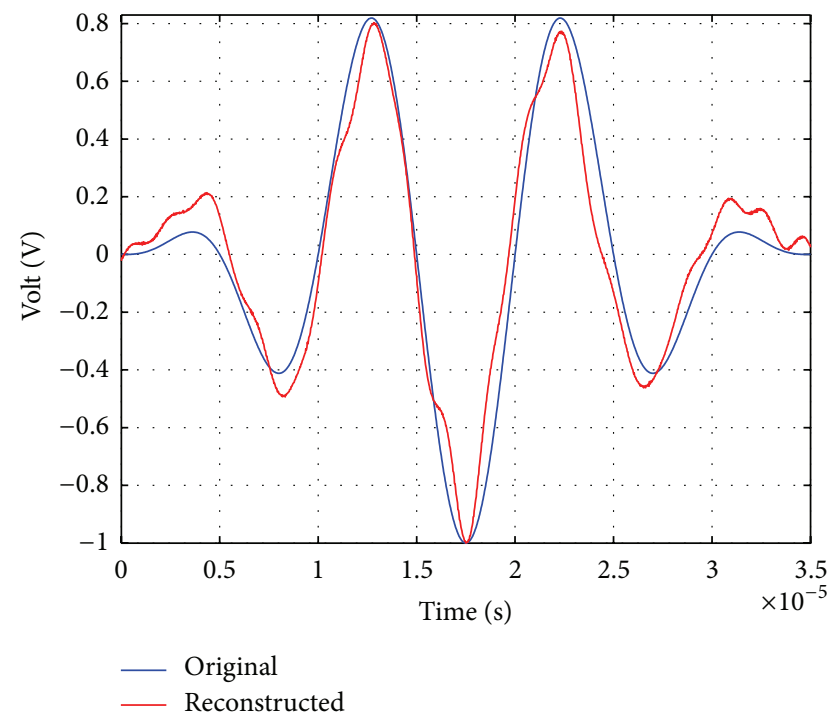

(b)

Figure 5: Original tone burst and reconstructed tone burst after time reversal procedure (path P7-16): (a) $50 \mathrm{~V}$ excitation and (b) $150 \mathrm{~V}$ excitation. Blue line: original and red line: reconstructed.

If $k_{\mathrm{B}}(\omega)=k_{\mathrm{A}}(\omega)$ when the same transducers are used for actuator and sensor, then

$$
V_{\mathrm{B}}(\omega)=G(\omega) V_{\mathrm{A}}(\omega) .
$$

When $V_{\mathrm{B}}(t)$ is time reversed in time domain and applied to transducer $B$ following the same procedure, a wave signal at transducer A is captured:

$$
V_{\mathrm{A}}^{\prime}(\omega)=G^{2}(\omega) V_{\mathrm{A}}(\omega) .
$$

If there is no defect along the wave propagation path between transducers $\mathrm{A}$ and $\mathrm{B}$, then, theoretically, $V_{\mathrm{A}}^{\prime}(t)$ is similar to $V_{\mathrm{A}}(t)$ in the time domain, and the shape of the reconstructed signal $V_{\mathrm{A}}^{\prime}(t)$ should be the same as the original input signal $V_{\mathrm{A}}(t)$ after normalization.

But the time reversal process is complicated owing to the multimodal characteristics of Lamb waves. When PZT $\mathrm{A}$ is excited with a tone burst signal, multimode signals are received by PZT B. In Figure 1, the narrowband input frequency is selected so that only the first symmetric $S_{0}$ and antisymmetric $A_{0}$ modes are generated. When the response signal is reversed in the time domain and reemitted to PZT $\mathrm{B}$, each signal associated with the $A_{0}$ or $S_{0}$ mode at PZT B creates both $S_{0}$ and $A_{0}$ modes producing a total of four modes in the reconstructed signal. After superposition of signals, the reconstructed signal consists of the main packets in the middle and two side packets around the main mode wave packet. Finally, the shape of the main packets will be practically the same as the original input signal.

Because the time reversibility of waves is fundamentally based on the linear reciprocity of the system $[7,8]$, the linear reciprocity and the time reversibility break down if there exists any source of wave distortion due to wave scattering along the wave path. A nonlinear scatterer in the wave path results in a modification in the wave's central frequency so that $G(\omega)$ is no longer a scalar, and the shape of the reconstructed $V_{\mathrm{A}}^{\prime}(t)$ after wave interaction with damage will differ from that of wave due to wave distortion. With the original wave signal $V_{\mathrm{A}}(t)$ and the reconstructed wave signal $V_{\mathrm{A}}^{\prime}(t)$ after time reversal in the time domain, a damage index (DI) can be defined by the difference between the two signals. Hence, nonlinear type damages such as closed cracks in metallic structures and delaminations in composite structures could be detected by comparing the discrepancy between the original input signal and the reconstructed signal.

\section{Damage Index and Imaging Method}

The damage index can be defined using the characteristics of wave signals in the time domain. In the time domain, the differences between the original incident wave signal $V_{\mathrm{A}}(t)=\left\{v_{1}, v_{2}, \ldots, v_{n}\right\}$ and the reconstructed signal $V_{\mathrm{A}}^{\prime}(t)=$ $\left\{v_{1}^{\prime}, v_{2}^{\prime}, \ldots, v_{n}^{\prime}\right\}$ of each actuator-sensor path can be measured quantitatively by the correlation coefficient, defined as

$$
\rho_{v_{i}, v_{i}^{\prime}}(t)=\frac{n \sum v_{i} v_{i}^{\prime}-\sum v_{i} \sum v_{i}^{\prime}}{\sqrt{\left[n \sum v_{i}-\left(\sum v_{i}\right)^{2}\right]\left[n \sum v_{i}^{\prime}-\left(\sum v_{i}^{\prime}\right)^{2}\right]}} .
$$

Before correlation, both $V_{\mathrm{A}}(t)$ and $V_{\mathrm{A}}^{\prime}(t)$ are normalized. The value of $\rho_{v_{i}, v_{i}^{\prime}}(t)$ defines the degree of similarity between the two signals $V_{\mathrm{A}}(t)$ and $V_{\mathrm{A}}^{\prime}(t)$. When each signal is perfectly approximated by the other, $\rho_{v_{i}, v_{i}^{\prime}}(t)=1$. The damage index (DI) is defined as

$$
\mathrm{DI}=1-\rho_{v_{i}, v_{i}^{\prime}}(t) .
$$

In this way, the higher the DI, the higher the possibility of the existence of damage or the closer the damage to the wave 


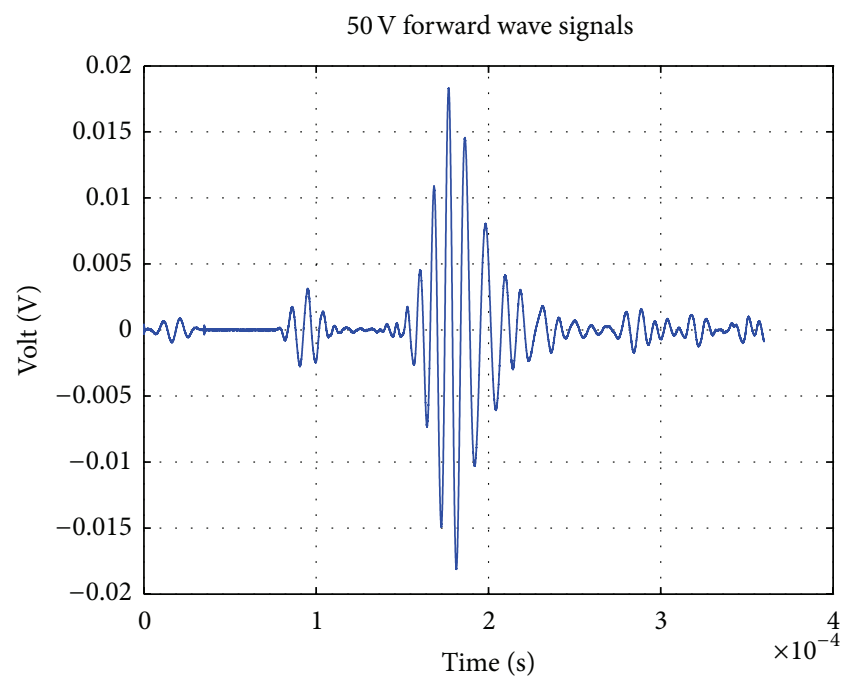

(a)

$50 \mathrm{~V}$ TR response signals

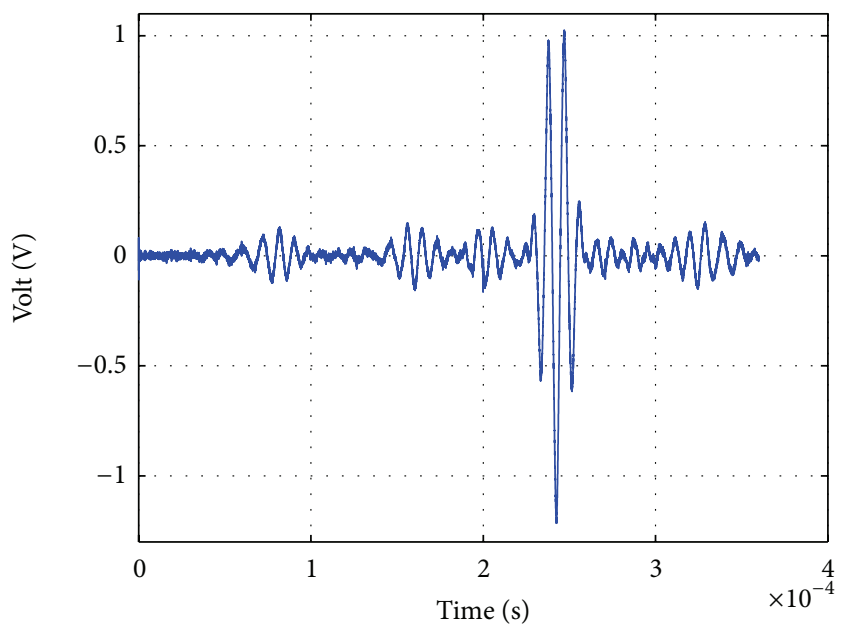

(c)

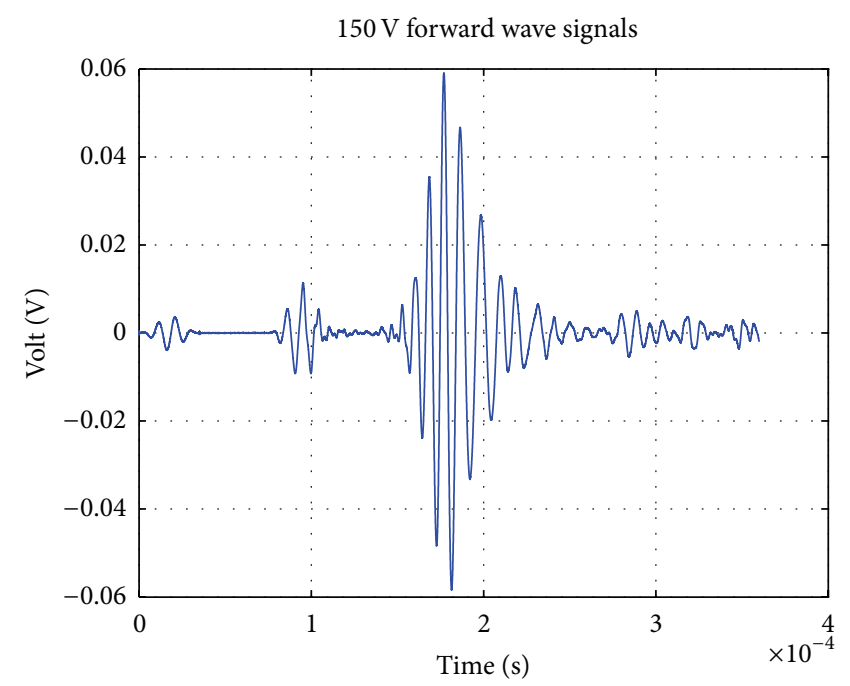

(b)

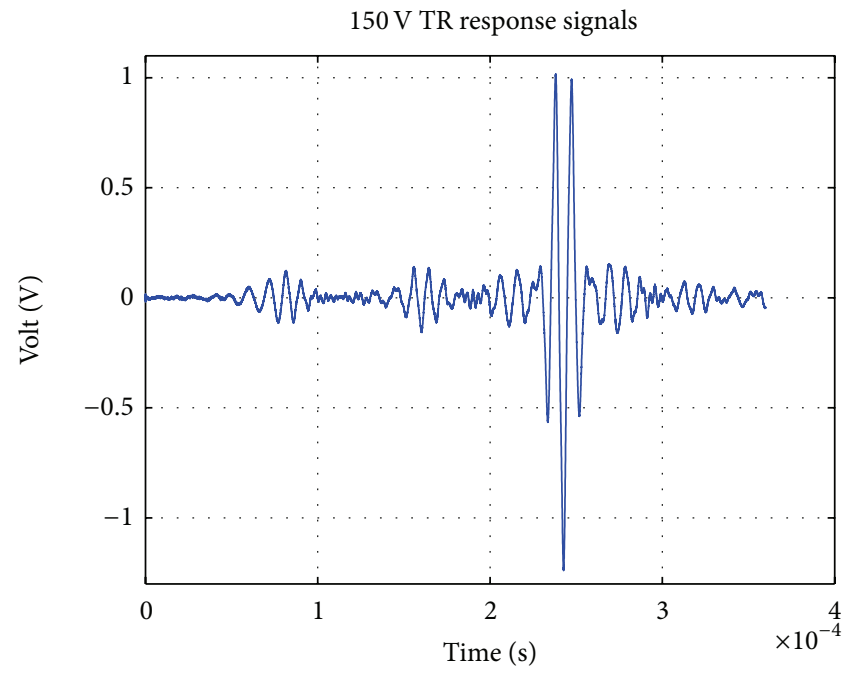

(d)

FIGURE 6: The receive waves of Lamb wave time reversal procedure for the path P6-12: (a) forward wave after $420 \mathrm{~mm}$ (50 V excitation), (b) forward wave after $420 \mathrm{~mm}$ ( $150 \mathrm{~V}$ excitation), (c) reconstructed waves (50 V excitation), and (d) reconstructed waves (150 V excitation).

path, as defined by the time reversibility of the input wave signal.

With the existence of damage at a location near the wave path, the effect of the DI is confined with a normal distribution function, which means that the effect of the DI is maximized on the wave path and decreases gradually as the distance from the wave path increases [9]:

$$
f(d)=\frac{1}{\sigma \sqrt{2 \pi}} e^{-(d-\mu)^{2} / 2 \sigma^{2}} \text { for }-\infty<d<\infty
$$

In (7), $\mu$ and $\sigma$ were set to 0 and $40 \mathrm{~mm}$, respectively. The value of $d$ was determined in an elliptical zone for each sensing path as $d=\left(D_{a}+D_{s}\right) / D_{k}-1$, where $D_{k}$ is the distance between the actuator and sensor for the $k$ th sensing path and $D_{a}$ and $D_{s}$ are the distances between a grid to the actuator and the sensor, respectively.
Assuming that there are $n$ sensing paths for damage identification from the sensor network, the probability of the presence of damage at position $(x, y)$ can be written as [10]

$$
P(x, y)=\sum_{k=1}^{n} P_{k(x, y)}=\sum_{k=1}^{n} \mathrm{DI}_{k} f_{k}(d),
$$

where $P_{k(x, y)}$ is the probability of the presence of damage at position $(x, y)$ for the $k$ th sensing path and $\mathrm{DI}_{k}$ and $f_{k}(d)$ are the damage index defined in (6) and (7) for the $k$ th sensing path and the effect of the damage index for the $k$ th sensing path on the presence of damage in the position, respectively.

This process was applied to each individual path, and $P(x, y)$ at each grid was obtained for the inspection area. By combining all the paths, the existence of damage was highlighted by an area with high values of $P(x, y)$. 


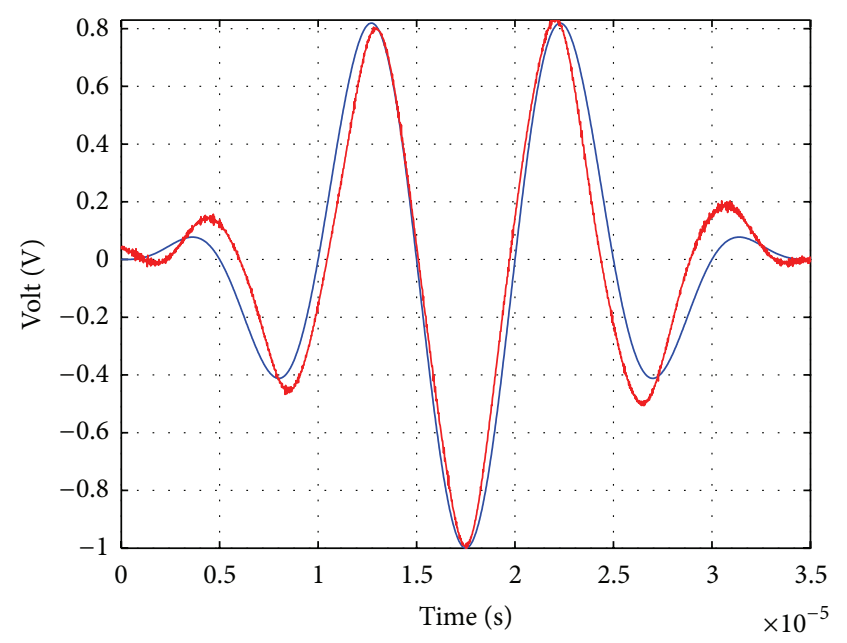

- Original

— Reconstructed

(a)

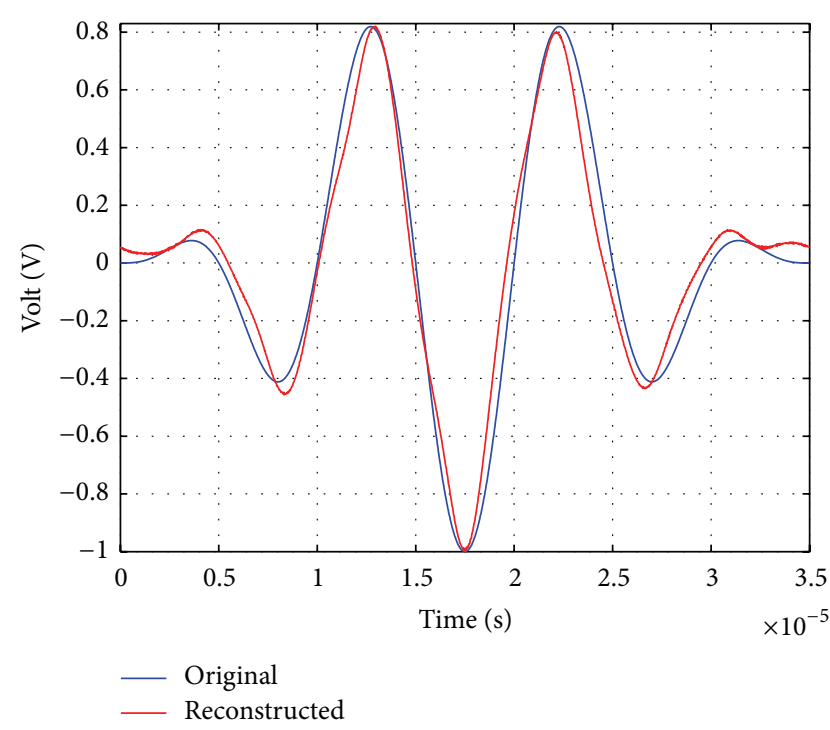

(b)

FIGURE 7: Original tone burst and reconstructed tone burst after time reversal procedure for path P6-12: (a) 50 V excitation, (b) 150 V excitation. Blue line: original and red line: reconstructed.

TABLE 1: Coordinates of transducers.

\begin{tabular}{|c|c|c|c|}
\hline \multicolumn{2}{|r|}{ Actuators } & \multicolumn{2}{|r|}{ Sensors } \\
\hline PZT & Coordinate $(x, y) / \mathrm{mm}$ & PZT & Coordinate $(x, y) / \mathrm{mm}$ \\
\hline 1 & $(290,290)$ & 9 & $(290,710)$ \\
\hline 2 & $(350,290)$ & 10 & $(350,710)$ \\
\hline 3 & $(410,290)$ & 11 & $(410,710)$ \\
\hline 4 & $(470,290)$ & 12 & $(470,710)$ \\
\hline 5 & $(530,290)$ & 13 & $(530,710)$ \\
\hline 6 & $(590,290)$ & 14 & $(590,710)$ \\
\hline 7 & $(650,290)$ & 15 & $(650,710)$ \\
\hline 8 & $(710,290)$ & 16 & $(710,710)$ \\
\hline
\end{tabular}

TABLE 2: DI of different paths.

\begin{tabular}{lccccc}
\hline Number & Path & DI & Number & Path & DI \\
\hline 1 & $2-10$ & 0.1982 & 9 & $5-14$ & 0.0499 \\
2 & $2-13$ & 0.0863 & 10 & $6-11$ & 0.0302 \\
3 & $3-9$ & 0.1451 & 11 & $7-9$ & 0.1188 \\
4 & $3-12$ & 0.0965 & 12 & $7-14$ & 0.0535 \\
5 & $4-12$ & 0.0628 & 13 & $7-16$ & 0.0345 \\
6 & $4-13$ & 0.0440 & 14 & $8-12$ & 0.0414 \\
7 & $4-16$ & 0.0459 & 15 & $8-13$ & 0.0420 \\
8 & $5-10$ & 0.1394 & 16 & $8-14$ & 0.0503 \\
\hline
\end{tabular}

\section{Experimental Study}

4.1. Experimental Setup. The overall test configuration of this study is shown in Figure 2. A $1000 \mathrm{~mm} * 1000 \mathrm{~mm} * 2 \mathrm{~mm}$ aluminum plate is adopted to evaluate the method presented in this paper. A sensor network is created consisting of 16 circular PZTs (APC 850) with a distance of $60 \mathrm{~mm}$ between the elements. Elements (P1 to P16), $6.35 \mathrm{~mm}$ in diameter and $0.25 \mathrm{~mm}$ in thickness, were surface mounted on the aluminum plate. Their positions are listed in Table 1. A steel block with the diameter and height of $15 \mathrm{~mm}$ and $30 \mathrm{~mm}$ is attached on the aluminum plate to simulate the linear scatterer at location $(500,500) \mathrm{mm}$. A sandpaper polished bolt is tightened on the aluminum plate apart from the center at location $(380,440) \mathrm{mm}$ by a torque wrench with torque of $19 \mathrm{~N} \cdot \mathrm{m}$, simulating the closed crack (Figure 2(a)). An arbitrary-function generator (Agilent33522A) and a highvoltage amplifier (TEGAM2350) are used to control the signal transmitted and an oscilloscope (Agilent D50-X3014A) is used for recording the sensing signals. The geometry of the plate with the PZT array and damage positions is shown in Figure 3.

Park et al. [4] discussed the effect of boundary reflections of waves on the time reversal process (TRP). The numerical simulations and experimental tests indicated that the boundary reflection had no substantial effect on the reconstructed wave signal. Through studying a free boundary and with wave-absorbing material around the edges, Song et al. [10] found that the main wave pack was almost the same and the side bands differ slightly after the time reversal process was applied. In this paper, the reflections from structural boundaries are not considered by choosing appropriate time window in the wave signals.

Because of the characteristics of the closed crack, with low amplitude voltage excitation that could not overcome the threshold, the closed crack behaves as a linear scatterer. While high amplitude voltage is applied, once the threshold of the closed crack is overcome, the closed crack can be opened and the nonlinear response is produced. The experiment is conducted as follows. A 3.5 counts $100 \mathrm{kHz}$ Hanning window 


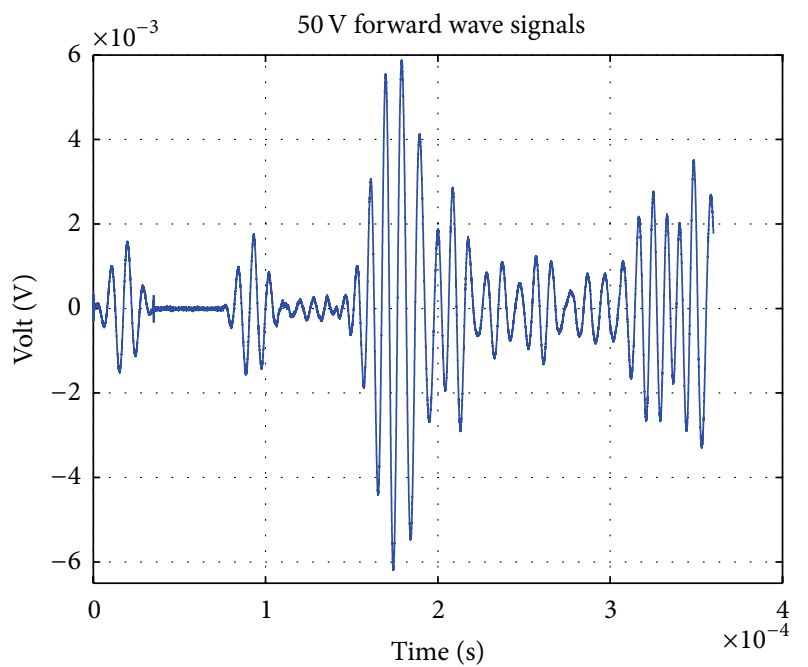

(a)

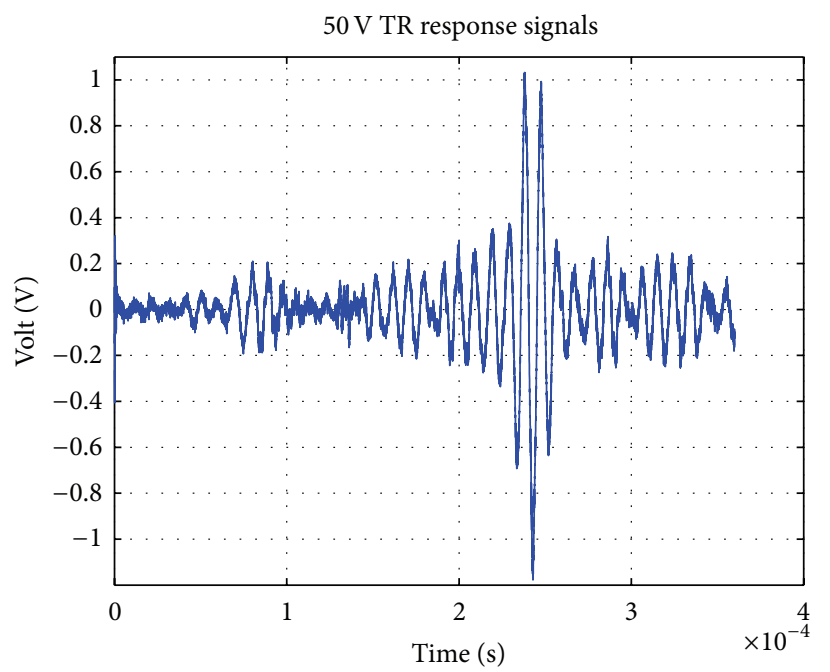

(c)

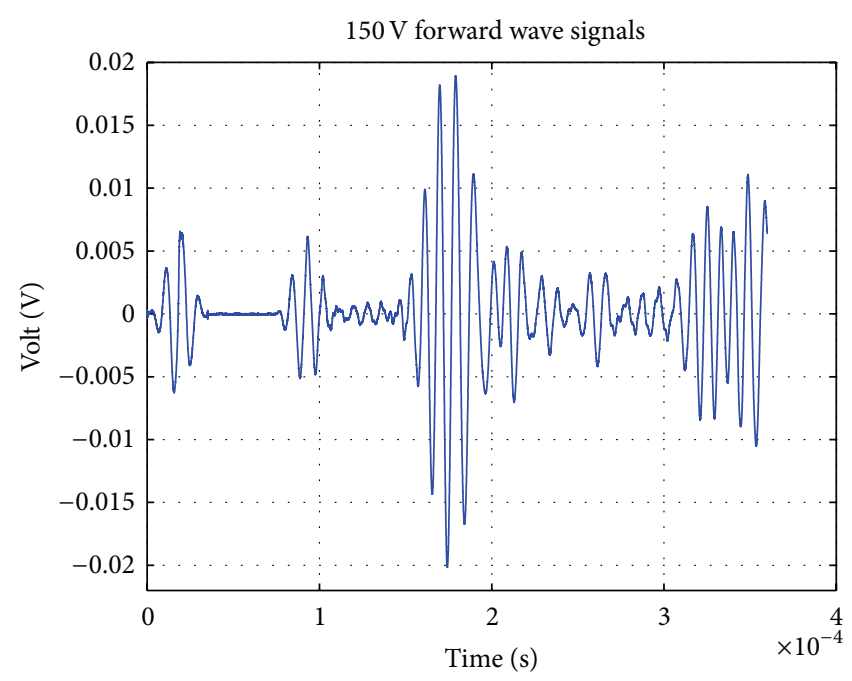

(b)

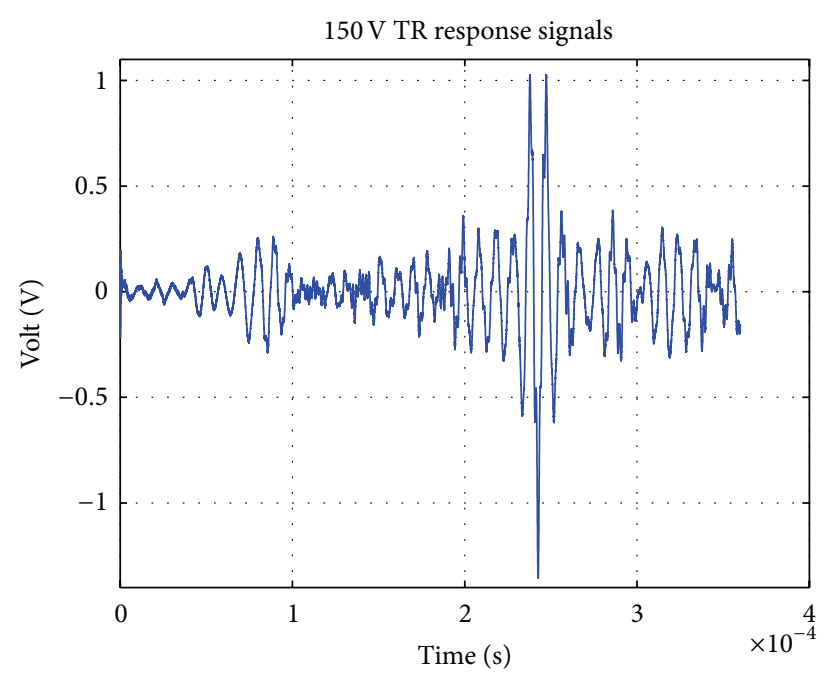

(d)

FIGURE 8: Experimental response of Lamb wave time reversal procedure for path P2-11: (a) forward wave after $424 \mathrm{~mm}$ (50 V excitation), (b) forward wave after $424 \mathrm{~mm}$ (150 V excitation), (c) reconstructed waves (50 V excitation), and (d) reconstructed waves (150 V excitation).

modulated sine tone burst is used as the excitation signal. First, an amplitude of $50 \mathrm{~V}$ peak-to-peak excitation is chosen to actuate one row of PZT transducers (number 1-number 8), one at a time. The eight transducers (number 9-number 16) record the signals. Each recorded signal is time reversed by using appropriate time window. Before reemitting, the timereversed signals are normalized to the same amplitude, so that the contribution of the backpropagated field is approximately the same from each transducer; the backpropagated wave field is recorded in the receiver positions and analyzed. Then, a larger amplitude of $150 \mathrm{~V}$ peak-to-peak excitation is applied with the same above steps to record the responses.

4.2. Experimental Results. In this study, signals extracted from three paths (P7-16, P6-12, and P2-12) in the aluminum plate are selected for demonstration. Figure 4 shows the experimental results for the time reversal of the Lamb wave on the plate of the path P7-16 with $50 \mathrm{~V}$ peak-to-peak excitation and $150 \mathrm{~V}$ peak-to-peak excitation. This path is away from the block and the bolt. The forward waves are captured after propagation of $424 \mathrm{~mm}$ by PZT16 (Figures 4(a), 4(b)). (Note: the first wave packet shown in the experimental forward wave is the E/M coupling.) Figures 4(c) and 4(d) show the reconstructed signals which are normalized. The main packets of the reconstructed and the original tone burst signals are shown in Figures 5(a) and 5(b). The reconstructed signals with low amplitude and high amplitude of excitation all achieved a similarity of $98 \%$ to the original tone burst signals (according to (6)). It indicates that this path is not influenced by the closed crack and linear scatterer and it can be treated as intact path.

The experiment results of the path P6-12 for the time reversal of the Lamb wave propagating are shown in Figure 6. The block is in this path and the bolt is away from it. 


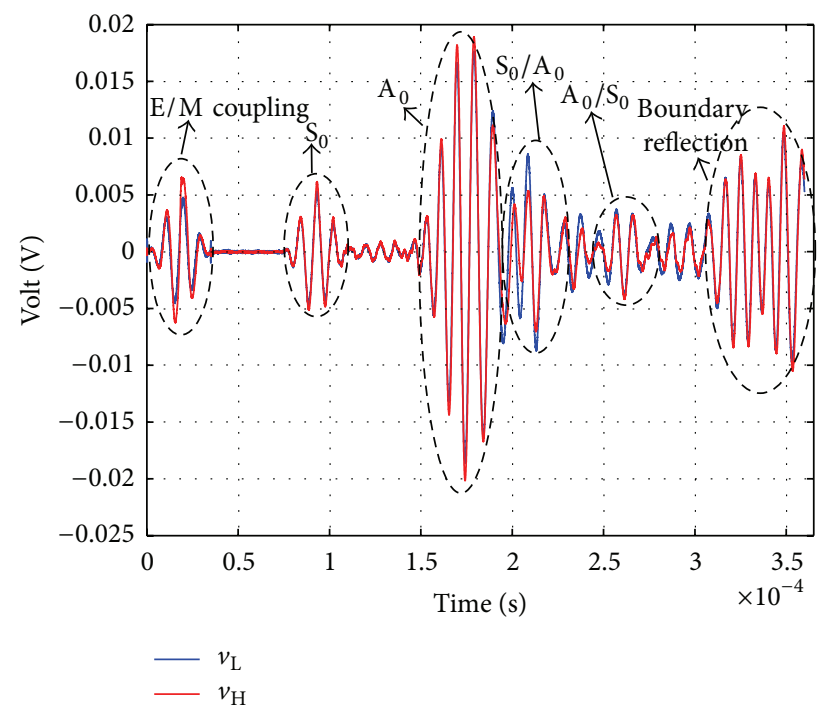

FIGURE 9: Baseline signal $v_{L}$ (50 V excitation) and the current signal $v_{H}(150 \mathrm{~V}$ excitation).

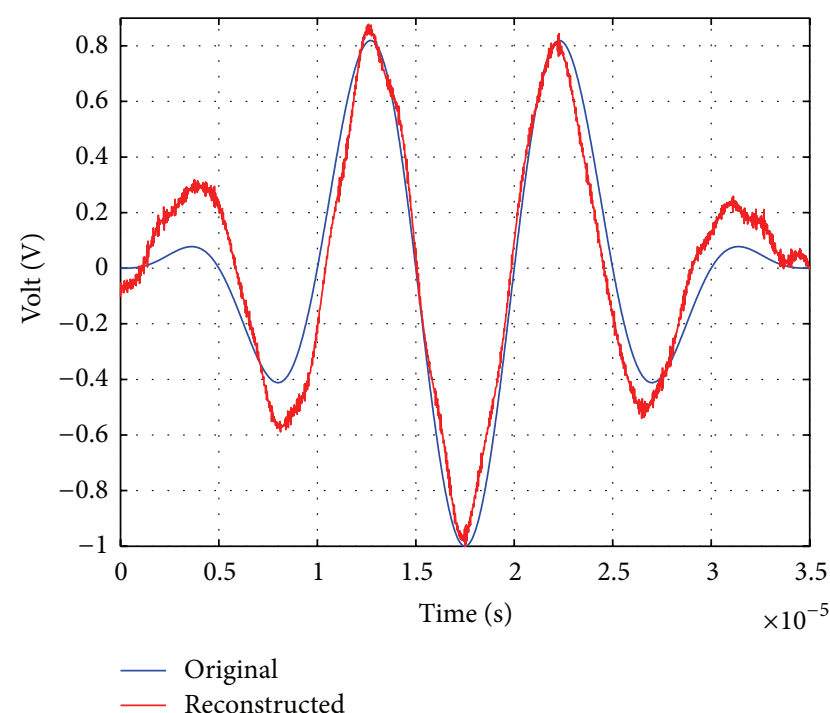

(a)

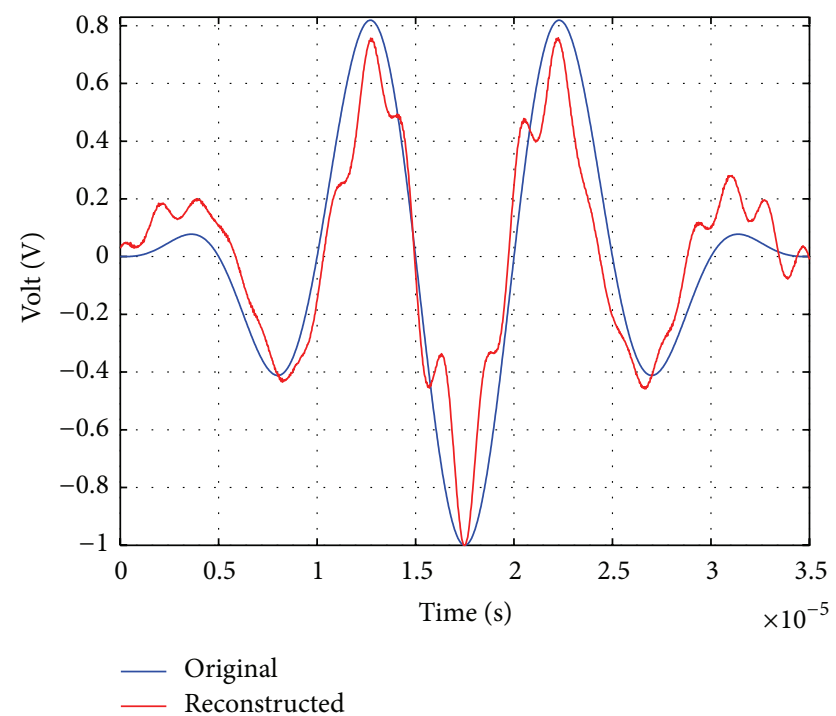

(b)

FIGURE 10: Original tone burst and reconstructed tone burst of path P2-11 after time reversal procedure: (a) $50 \mathrm{~V}$ excitation and (b) $150 \mathrm{~V}$ excitation: Blue line: original and red line: reconstructed.

Figures 6(a) and 6(b) show the waves captured after propagation of $420 \mathrm{~mm}$ by PZT12 with low and high excitations, respectively. The reconstructed waves are shown in Figures 6(c) and 6(d); the main wave packets in the reconstructed wave resemble well the original tone burst. Figures 7(a) and 7(b) show the reconstructed and the original tone burst signals for the two excitations; the similarity of the reconstructed signals to the original tone burst signals is the same as the intact path (P7-16); it indicates that the linear scatterers had not broken down the linear reciprocity and the time reversibility; the existence of linear damage would not influence the detection of closed crack.
The bolt (closed crack) is on the wave path P2-11 and the low amplitude voltage excitation $(50 \mathrm{~V})$ and the high amplitude voltage excitation $(150 \mathrm{~V})$ experimental results for the time reversal of the Lamb wave are, respectively, shown in Figure 8. Figures $8(a)$ and $8(b)$ show the forward wave captured after propagation of $424 \mathrm{~mm}$ by PZT11. The reconstructed waves of $50 \mathrm{~V}$ and $150 \mathrm{~V}$ excitations are shown in Figures 8(c) and 8(d), respectively.

In order to extract the nonlinear contributions from the received signal of the path $\mathrm{P} 2-11$ with $150 \mathrm{~V}$ excitation, we need to quantify the difference between the received signal and a linear reference signal. The linear reference signal is 


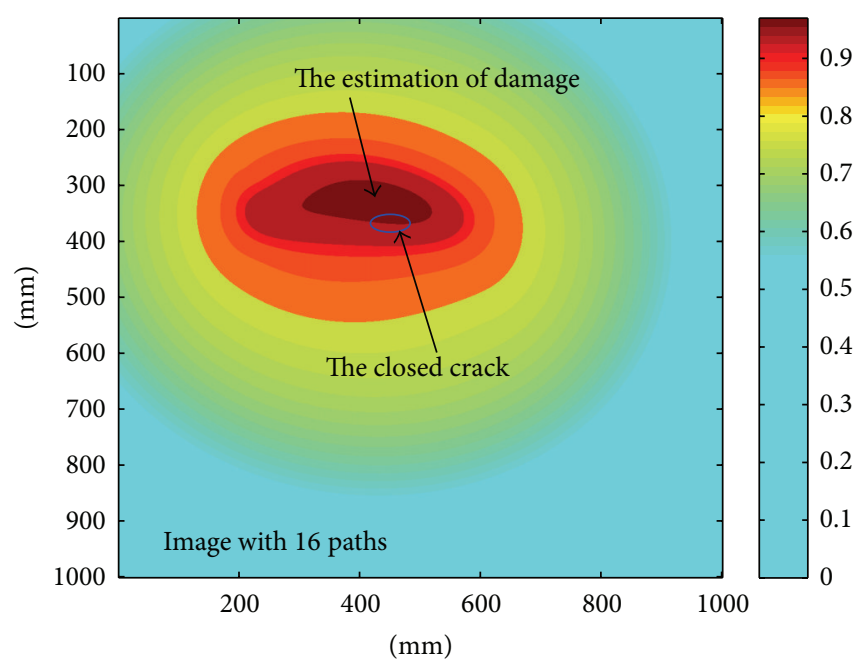

(a)

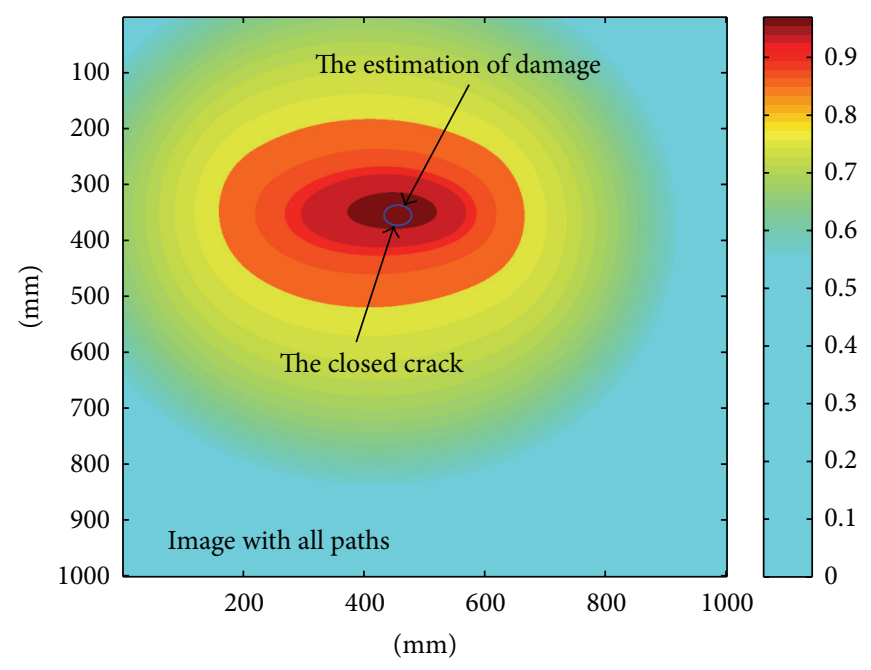

(b)

FIGURE 11: Image of damage constructed with (a) 16 actuator-sensor paths and (b) 64 actuator-sensor paths; the circle indicates the closed crack; the arrow indicates the estimation of damage.

often hard to be defined in practice due to the character of the closed crack; the response signals of low amplitude ( $50 \mathrm{~V}$ excitation), which could not open the closed crack, are collected by PZT11 as the instantaneous baseline. Then, the response signals of $150 \mathrm{~V}$ peak-to-peak excitation, which can open the closed crack, are recorded as the current signal. The current signal $v_{H}$ and the baseline $v_{L}$ of path P2-11 are shown in Figure 9 . Note that $v_{L}$ has been multiplied by 3 . The reconstructed and the original tone burst signals with $50 \mathrm{~V}$ excitation and $150 \mathrm{~V}$ excitation are shown in Figures 10(a) and 10 (b), respectively. Figure 10 indicates that the closed crack is simulated successfully with the bolt and the existence of closed crack breaks down the linear reciprocity and the time reversibility; the produced nonlinear response significantly influences reconstruction.

From the network of 16 PZT elements, a total of 128 paths were available, but with the dual function of the PZT elements, they could be reduced to 64 paths. Different groups of sensing paths are thus used to locate the closed crack. The correlation coefficients between the original and the reconstructed signals are calculated, and the DI is obtained using (6). 16 random paths are selected and shown in Table 2. Estimation of the presence of damage in the aluminum plate is obtained. Figure 11 illustrates the images of different group paths, where the circle indicates the exact location of the closed crack, and the arrow highlights the estimation of damage. The values in each image were normalized. Overall, the results demonstrate that the proposed method could identify and locate the damage clearly.

\section{Conclusion}

In this paper, a damage detection method using nonlinear ultrasonic time reversal process is proposed to detect and locate closed crack in aluminum plate structures. The closed crack is simulated by a bolt with moderate clamp torque and the nonlinear effects are generated by contacts between bolt surface and aluminum plate under excitation voltage. The damage index defined using the correlation coefficient between the excitation wave signal and the reconstructed wave signal after time reversal is used for damage identification and imaging. Experimental studies are carried out to examine the proposed method. The results of tests demonstrate that the simulated closed crack using the bolt could represent the crack damage in actual structures and the time reversal imaging algorithm has potential to locate nonlinear scatterers in real structures with simple PZT arrays, and this approach is not influenced by linear scatterers in the wave paths.

\section{Disclosure}

The authors warrant that the paper is submitted for first publication in the journal and that is not being considered for publication elsewhere. The authors moreover warrant that they have obtained all necessary permissions for the reproduction of any copyright works.

\section{Acknowledgments}

This research is funded by the National Science Foundation of China under Contract nos. 51078293 and 51378402.

\section{References}

[1] K. E.-A. Van Den Abeele, P. A. Johnson, and A. Sutin, "Nonlinear Elastic Wave Spectroscopy (NEWS) techniques to discern material damage, Part I: nonlinear wave modulation spectroscopy (NWMS)," Research in Nondestructive Evaluation, vol. 12, no. 1, pp. 17-30, 2000.

[2] R. K. Ing and M. Fink, “Time-reversed lamb waves," IEEE Transactions on Ultrasonics, Ferroelectrics, and Frequency Control, vol. 45, no. 4, pp. 1032-1043, 1998. 
[3] R. K. Ing and M. Fink, "Time recompression of dispersive lamb waves using a time reversal mirror-application to flaw detection in thin plates," in Proceedings of the IEEE Ultrasonics Symposium, pp. 659-663, November 1996.

[4] H. W. Park, S. B. Kim, and H. Sohn, "Understanding a time reversal process in Lamb wave propagation," Wave Motion, vol. 46, no. 7, pp. 451-467, 2009.

[5] B. Xu and V. Giurgiutiu, "Single mode tuning effects on lamb wave time reversal with piezoelectric wafer active sensors for structural health monitoring," Journal of Nondestructive Evaluation, vol. 26, no. 2-4, pp. 123-134, 2007.

[6] T. J. Ulrich, P. A. Johnson, and A. Sutin, "Imaging nonlinear scatterers applying the time reversal mirror," Journal of the Acoustical Society of America, vol. 119, no. 3, pp. 1514-1518, 2006.

[7] G. Draeger, D. Cassereau, and M. Fink, "Theory of the timereversal process in solids," Journal of the Acoustical Society of America, vol. 102, no. 3, pp. 1289-1295, 1997.

[8] M. Fink and C. Prada, "Acoustic time-reversal mirrors," Inverse Problems, vol. 17, no. 1, pp. R1-R38, 2001.

[9] D. Wang, L. Ye, and Y. Lu, "A probabilistic diagnostic algorithm for identification of multiple notches using digital damage fingerprints (DDFs)," Journal of Intelligent Material Systems and Structures, vol. 20, no. 12, pp. 1439-1450, 2009.

[10] S. Mustapha, L. Ye, D. Wang, and Y. Lu, "Debonding detection in composite sandwich structures based on guided waves," AIAA Journal, vol. 50, no. 8, pp. 1697-1706, 2012. 

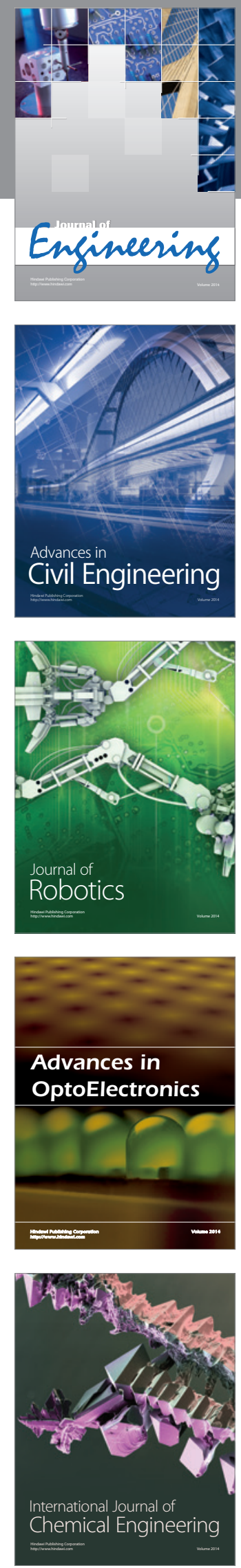

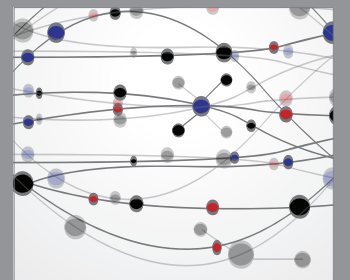

The Scientific World Journal
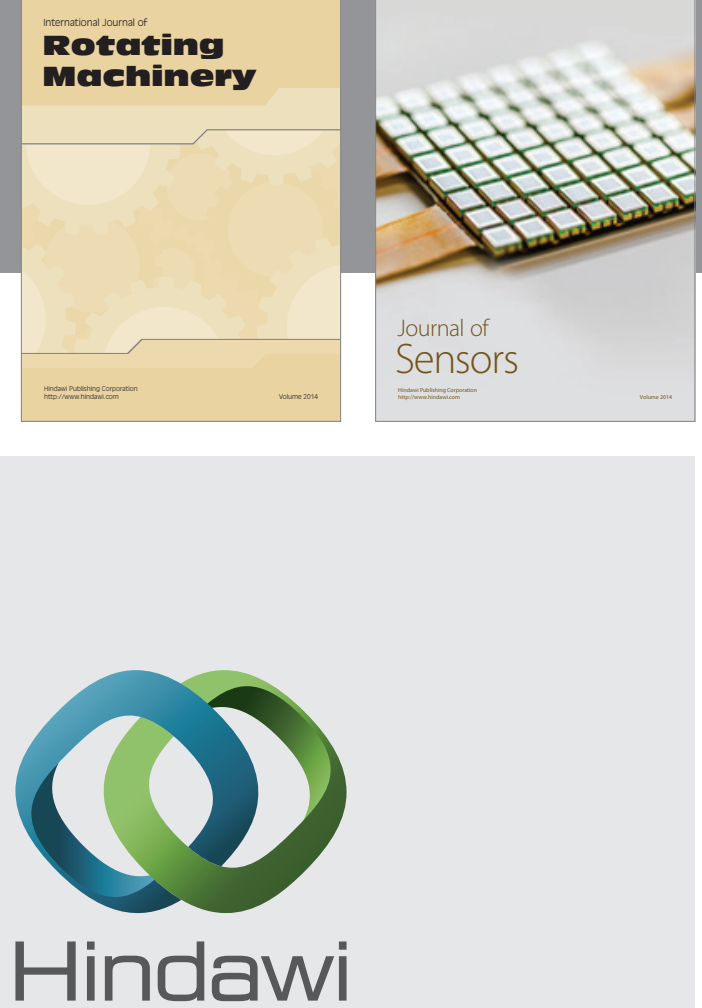

Submit your manuscripts at http://www.hindawi.com
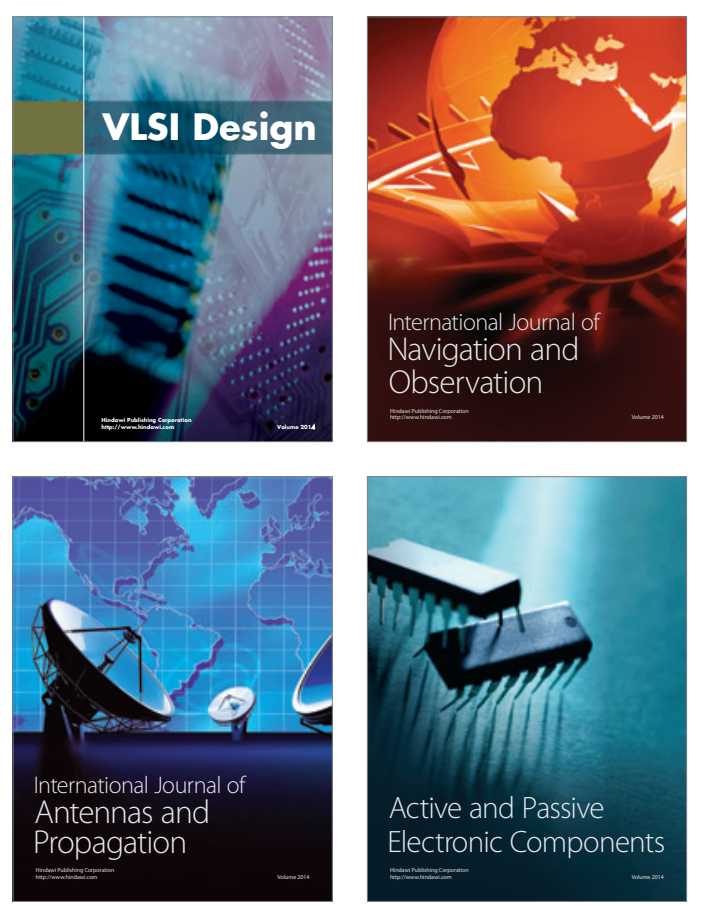
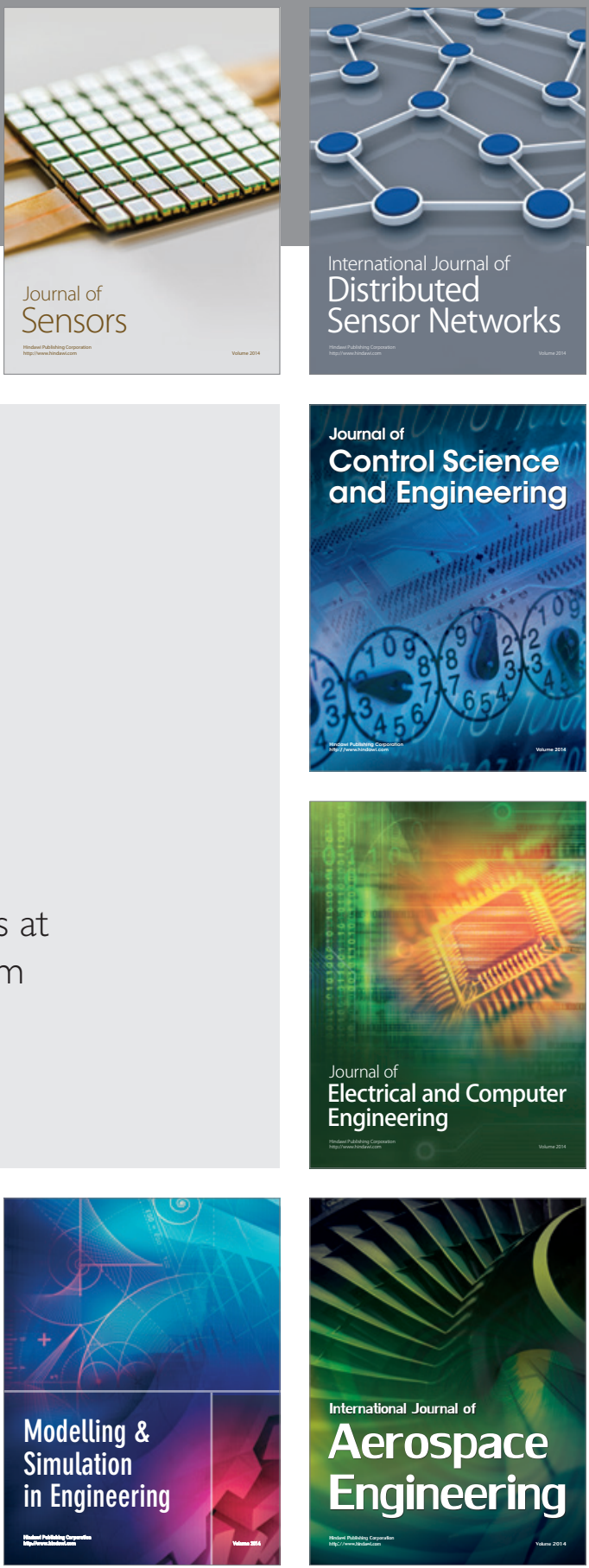

Journal of

Control Science

and Engineering
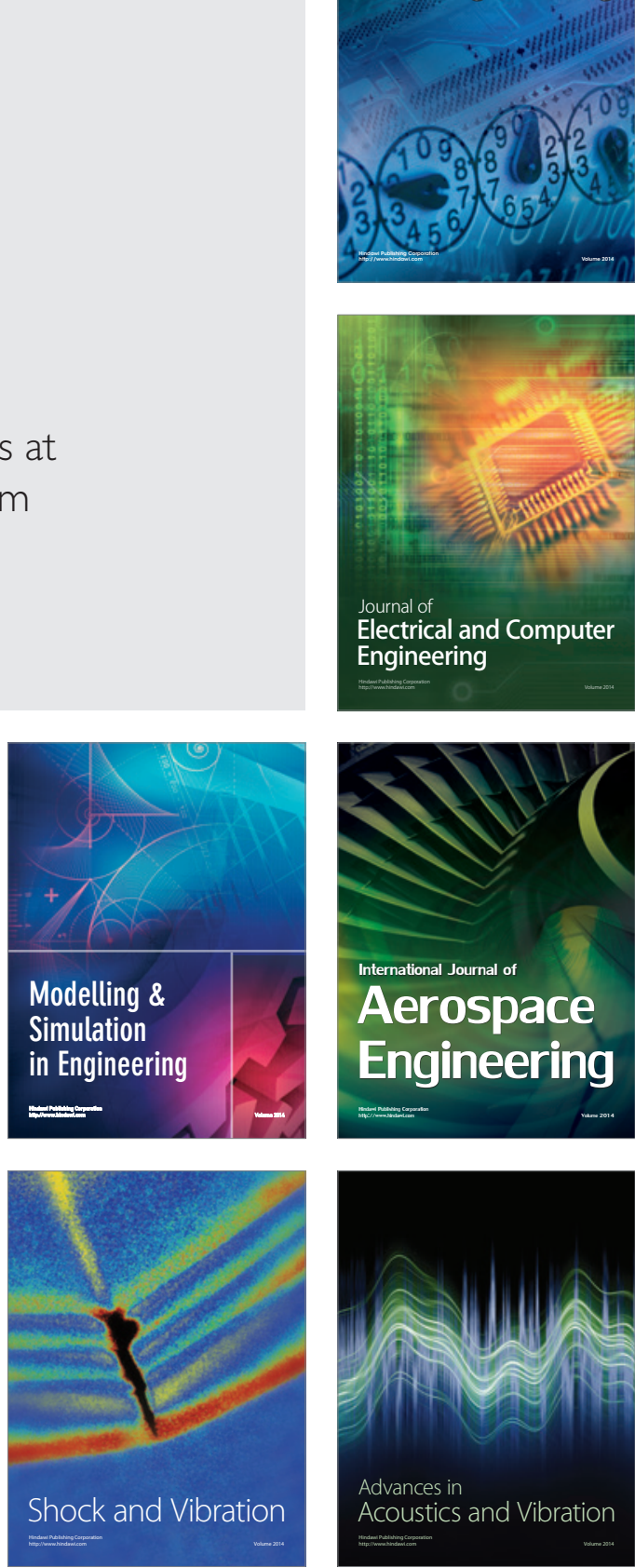